\section{Biogenic silver nanoparticles embedded polyvinyl alcohol nanofibrous scaffolds avert tumour and bacterial growth}

\author{
G. U. Preethi ${ }^{1}$, B. S. Unnikrishnan ${ }^{1}$, \\ Manu M. Joseph ${ }^{1,2}$, R. Shiji ${ }^{1}$ and \\ T. T. Sreelekha ${ }^{1, *}$ \\ ${ }^{1}$ Laboratory of Biopharmaceuticals and Nanomedicine, \\ Division of Cancer Research, Regional Cancer Centre, \\ Thiruvananthapuram 695 019, India \\ ${ }^{2}$ Chemical Sciences and Technology Division, \\ Organic Chemistry Section, CSIR-National Institute for \\ Interdisciplinary Science and Technology, \\ Thiruvananthapuram 695 019, India
}

This communication describes a carefully designed strategy which blends biogenic silver nanoparticles with PVA scaffolds to fabricate SNP@PVA electrospun scaffolds with admirable physico-chemical properties. The hybrid scaffold demonstrated cyto-compatibility and hemo-compatibility with no adverse effects on the surrounding cells, as demonstrated by multiple assays. The growth of cancer cells was greatly prevented by SNP@PVA scaffolds, while allowing growth of normal cells. The outstanding antimicrobial features of the scaffold can be attributed to the presence of silver nanoparticles and proves the use of SNP@PVA for biomedical applications.

Keywords: Cancer cells, electrospinning, nanofibrous scaffold, polyvinyl alcohol, silver nanoparticles.

MULTIDISCIPLINARY approaches engaging nanofibrous scaffolds (NS) attract attention in biomedicine. NS are composed of fibres of several nanometre to micrometre diameter, similar to collagen, elastin and keratin fibres of the extracellular matrix (ECM), and help in cell attachment, proliferation and differentiation ${ }^{1}$. Electrospinning has been extensively used as a simple and versatile technique to produce micro- and nanofibres of polymers, because it provides an effective way to fabricate continuous nanofibres with different structural designs and applications, from electronics to biomedicine. Woundhealing is a dynamic and complex physiological process, wherein the quest for an ideal dressing material is largely unmet so far. However, it is widely believed that a warm and moist milieu boosts healing and hence most of the wound-care products address these parameters. Thus arises the need for biocompatible wound-dressing materials, and hence NS could be an effective choice.

Polysaccharides should be considered as a unique source of natural compounds. They present an enormous variety of structures, but are still underexploited. Owing

\footnotetext{
*For correspondence. (e-mail: sreelekhatt@rcctvm.gov.in)
}

to their many unique physico-chemical features, the biological activities of polysaccharides have attracted more attention in biomedicine. Tamarindus indica seed kernel is a rich source of polysaccharides. It is predominantly composed of galactoxyloglucan with a $(1 \rightarrow 4)$ - $\beta$-D-glucan backbone ${ }^{2,3}$, and displays a wide range of properties such as biocompatibility, biodegradability, high viscosity, $\mathrm{pH}$ tolerance, thermal stability and adhesiveness. Galactoxyloglucan (PST001) was isolated and extensively characterized in our laboratory; it was found to have anticancer and immunomodulatory properties ${ }^{4}$, both in vitro and in vivo. PST001 was effectively used both as a reducing and capping agent in the fabrication of biogenic silver nanoparticles (SNP@PST), which not only demonstrated cancer-targeted cytotoxicity but also proved to be an excellent antimicrobial agent ${ }^{5}$. Biogenic synthesis of metal nanoparticles helps reduce the toxicity and enhances biocompatibility.

A few synthetic polymers such as polylactic acid (PLA), polyvinyl alcohol (PVA), polyhydroxy alkanoates (PHA), polyglycolic acid (PGA) and polycaprolactone (PCL) come into play as promising candidates in the fabrication of scaffolds. Among these, PVA is a semicrystalline, highly hydrophilic, nontoxic and biocompatible polymer with excellent properties such as strength, permeability and thermal characteristics. Moreover, reports regarding the electrospinnability of PVA makes it an ideal candidate for the fabrication of scaffolds ${ }^{6}$.

Cancer is a heterogeneous fatal clinical abnormality with high complexity in nature, characterized with abnormal proliferation of cells and accounts for the second leading cause of death. Since wound-healing is an important factor for cancer patients, development of a wound healing scaffold with biocompatibility holds great importance. The present study deals with the fabrication of a PVA scaffold doped with silver nanoparticles using the anticancer polysaccharide PST001 by adopting the principles of green chemistry ${ }^{7}$, and to evaluate its potential as an antimicrobial wound-healing scaffold. Although prospective studies are warranted, the present study will enable further biomedical exploration of non-toxic biogenic polysaccharides.

PVA solutions (7\%) were prepared by dissolving PVA powder (PVA - fully hydrolysed, Alfa Aesar, Massachusetts, USA) in $50 \%$ acetic acid at $80^{\circ} \mathrm{C}$ with vigorous stirring for $4 \mathrm{~h}$ after the solution was cooled to room temperature. The acidified aqueous PVA solution was poured into a $5 \mathrm{ml}$ syringe fitted with $24 \times 1$ bluntend stainless steel needle of $0.5 \mathrm{~mm}$ inner diameter. Electrospinning of the PVA solution was carried out in a nanofibre electrospinning unit (ESPIN-NANO, Physics Equipments, Chennai) with high voltage supply by charging the solution at $30 \mathrm{kV}$, and the distance between the needle tip and the collector was set to be $10 \mathrm{~cm}$. The PVA solution was dispensed at a flow rate of $1 \mathrm{ml} / \mathrm{h}$ and the electrospun PVA nanofibrous mat was collected onto 
an aluminum foil as a support attached on the grounded stainless-steel collector ${ }^{8}$.

PST001 was isolated from $T$. indica seed kernel, as previously reported ${ }^{4}$. Briefly, shade-dried kernels were powdered and petroleum ether-washed for 3 days. After filtration, the powder was dried and boiled to soften the kernel tissue. The supernatant was collected and ethanol precipitated. Precipitate dissolved in water was chloroform separated, dialysed for $48 \mathrm{~h}$ and concentrated and above steps were repeated. The sample was purified using high-performance liquid chromatography (HPLC, Shimadzu), lyophilized (CHRIST ALPHA 2-4 LD PLUS, Germany) and stored at $4^{\circ} \mathrm{C}$ for further analysis. Silver nanoparticles were prepared using a facile one-step approach by the reduction of silver nitrate $\left(\mathrm{AgNO}_{3}\right)$ using PST001, as previously reported ${ }^{5}$. Particles were characterized for purity, size and surface charge. Size and zeta potential were measured using dynamic light scattering (DLS/Horiba, Japan).

The as-prepared PVA scaffold was used as a matrix to incorporate SNP@PST to fabricate PVA@SNP scaffold. Electrospinning of SNP@PST $(1 \mathrm{mg} / \mathrm{ml})$ with standardized PVA solution was done while maintaining the same conditions standardized for PVA scaffolds. Electrospun nanofibres that were deposited on aluminum foils wrapped on the rotating collector were sputter-coated with gold for $45 \mathrm{~s}$ and analysed using a scanning electron microscope (JEOL model JSM-6390LV) at a voltage of $15 \mathrm{kV}$. The nanoscaffolds were analysed using FTIR spectroscopy (Thermo Nicolet Avatar 370, Madison, WI, USA) to confirm the crosslinking.

The effect of scaffolds on normal red blood cells (RBCs) was evaluated by hemolysis assay, as reported previously ${ }^{9}$. Freshly prepared $(4 \mathrm{ml})$ ACD-human blood (1:3) was diluted with $5 \mathrm{ml} 0.9 \%$ saline. The prepared scaffolds with different concentrations of gel were cut into small pieces and kept in normal saline for $30 \mathrm{~min}$ at room temperature. The diluted blood was added to each sample and incubated for $60 \mathrm{~min}$ at $37^{\circ} \mathrm{C}$. Positive control was prepared by adding $500 \mu \mathrm{l}$ of diluted blood to $4 \mathrm{ml}$ of distilled water, and negative controls were in saline. All the test samples were centrifuged at $3000 \mathrm{rpm}$ for $10 \mathrm{~min}$. Optical density (OD) was measured at $540 \mathrm{~nm}$ using ELISA plate reader $^{10}$. The haemolysis percentage was estimated using the following formula

$$
\begin{aligned}
& \text { Haemolysis }(\%)= \\
& \frac{\text { OD of test sample }- \text { OD of negative control }}{\text { OD of positive control-OD of negative control }} \times 100 \text {. }
\end{aligned}
$$

Absorbance of haemoglobin in supernatants was measured with a microplate reader at wavelength $\lambda=540 \mathrm{~nm}$. The experiments were performed after gaining sanction from the Human Ethical Committee, Regional Cancer Centre, Thiruvananthapuram. Percentage haemolysis values were calculated from three separate experiments. SEM was used to confirm absence of cell lysis.

The human cancer cell line A549 (lung adenocarcinoma) was obtained from American Type Culture Collection (Manassas, VA, USA). Human normal keratinocytes cell line $(\mathrm{HaCaT})$ was obtained from National Centre for Cell Science, Pune. Cells were maintained in DMEM with $10 \% \mathrm{FBS}$ and under an atmosphere of $5 \% \mathrm{CO}_{2}$ at $37^{\circ} \mathrm{C}$.

Human keratinocytes (HaCaT) were seeded in DMEM medium with a concentration $3 \times 10^{5}$ cells $/ \mathrm{ml}$ and kept overnight. The medium was discarded and $1.8 \%$ agar containing $0.01 \%$ neutral red and $10 \%$ FBS was added. Scaffolds were placed on the agar before its complete solidification. Nontoxic filter paper soaked in Triton $\mathrm{X}-100$ was used as positive control and non-toxic filter paper alone was used as negative control. The scaffolds were kept in the incubator for $24 \mathrm{~h}$ and observed for the presence of zone.

Indirect method was used for measuring the cytotoxicity of soluble components of the scaffolds. The extracts were collected in the cell-culture medium after $24 \mathrm{~h}$ of incubation (conditioned medium) and used for cytotoxicity assay. The growth inhibitory capacity was evaluated by 3-(4,5-dimethylthiazol-2-yl)-2,5-diphenyltetrazolium bromide (MTT) assay as previously reported ${ }^{11}$. The absorbance was measured at $\lambda=570 \mathrm{~nm}$ using a microplate spectrophotometer (BioTek, Power Wave XS) after incubation for 24, 48 and $72 \mathrm{~h}$.

Scaffolds were UV-sterilized for $15 \mathrm{~min}$ and washed in PBS and pre-soaked for $15 \mathrm{~min}$ in complete medium. Cells (10,000 numbers) were seeded directly onto the scaffolds using micro-mass seeding method, and incubated at $37^{\circ} \mathrm{C}$ and $5 \% \mathrm{CO}_{2}$. MTT assay was done at regular intervals.

Acridine orange-ethidium bromide (AO-EB) doublestaining was used as the method for live/dead staining. Indirect and direct contact method was used for this study as well. For indirect method, 5000 cells per well were seeded and leachates from the scaffolds were treated, and live/dead images were taken using FITC filter. For direct staining, scaffolds were seeded with 10,000 cells and after specific time intervals they were collected, washed and stained with AO-EB. Then the cells were detected under an inverted fluorescent microscope using a FITC filter (Olympus 1X51, Singapore).

The antibacterial activities of control and composite scaffolds were studied using agar disc diffusion method. Bacterial strains used were Escherichia coli (MTCC 40) and Bacillus pumilus (MTCC 1607). In this method the microbial species was grown on nutrient agar $(\mathrm{pH} 7)$. Disc-shaped samples of control and composite scaffolds with $5 \mathrm{~mm}$ diameter were prepared and subjected to the inhibition zone test. The samples were UV-sterilized for $15 \mathrm{~min}$ and subsequently placed on culture plates. The plates were incubated for $24 \mathrm{~h}$ at $37^{\circ} \mathrm{C}$. The relative antibacterial effect was found by measuring the clearing 

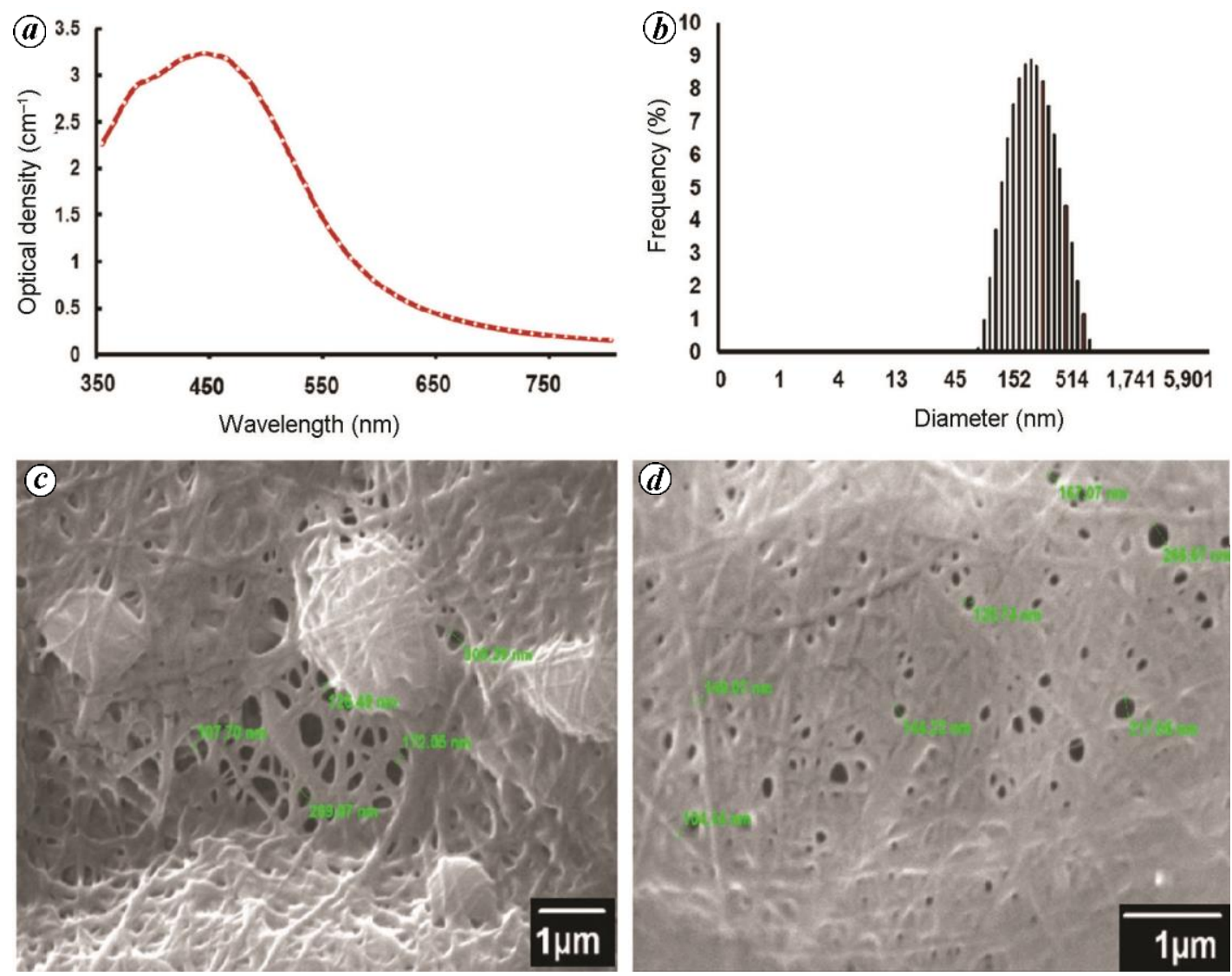

Figure 1. (a) UV-Visible spectra and (b) dynamic light scattering (DLS) of the selected SNP@PST. $\boldsymbol{c}, \boldsymbol{d}$, Scanning electron microscopic images of (c) polyvinyl alcohol (PVA) scaffold and (d) PVA@SNP scaffold.

zones of inhibition formed around the $\operatorname{discs}{ }^{11}$, using ciprofloxacin as a positive control.

Data were expressed as the mean \pm standard deviation (SD) of three replicates and were analysed using GraphPad PRISM software version 5.0 (GraphPad Software Inc., San Diego, CA, USA) ${ }^{5}$.

Electrospinning occurs when the applied electrical voltage exceeds a critical electrical potential at which the electrostatic force overcomes the surface tension of the polymer solution. Therefore, a low surface tension is desirable in electrospinning, as it reduces the critical voltage $\left(V_{c}\right)$ needed for the ejection of the jet from Taylor's cone $^{7}$. Different parameters were selected for the standardization of PVA scaffolds such as concentration, needle size, flow rate, voltage, collection distance, etc. (Supplementary Table 1). The surface tension of aqueous PVA solution exhibits a marked dependence on the degree of hydrolysis of PVA, which may result in an altered spinnability. We observed that $7.0 \mathrm{wt} \%$ is the minimum polymer concentration for fibre formation in this study. Below this critical value, application of voltage resulted in electrospraying or bead formation, primarily because of a Rayleigh instability ${ }^{7}$. The collection distance, temperature and solvent concentration were kept constant. Among the 16 combinations, scaffolds obtained by $7 \%$ PVA spun through $24 \times 1$-sized needles with a flow rate of $1 \mathrm{ml} / \mathrm{h}$ at $30 \mathrm{kV}$ voltage were found to be of optimum thickness for cell growth and hence selected for the downstream analysis (Supplementary Figure 1).

We found that PST001 successfully reduced $\mathrm{AgNO}_{3}$ to form silver nanoparticles with observable variation in colour. The successful production of SNP@PST was evaluated using UV-Vis spectroscopy, which produced the characteristic peak approximately around 420-430 nm (Figure $1 a$ ). PST001 not only acted as a reducing agent for the production of AgNPs but also served as a capping agent, imparting stability to the SNP@PST and preventing their reaggregation without the use of any other capping agent ${ }^{12}$. The nanoparticles were further screened for their hydrodynamic size and zeta potential using dynamic light scattering (DLS), which indicated that the particles have an average size of $241 \mathrm{~nm}$ (Figure $1 b$ ) and zeta -10 . If most of the particles in a colloid have large negative/positive zeta potential, then they will repel each other and there will be less chances of particles aggregation ${ }^{13}$.

After preparation of PVA scaffolds and SNP@PST, we incorporated the biogenic silver nanoparticles into the scaffold. The excellent stability, biocompatibility and 

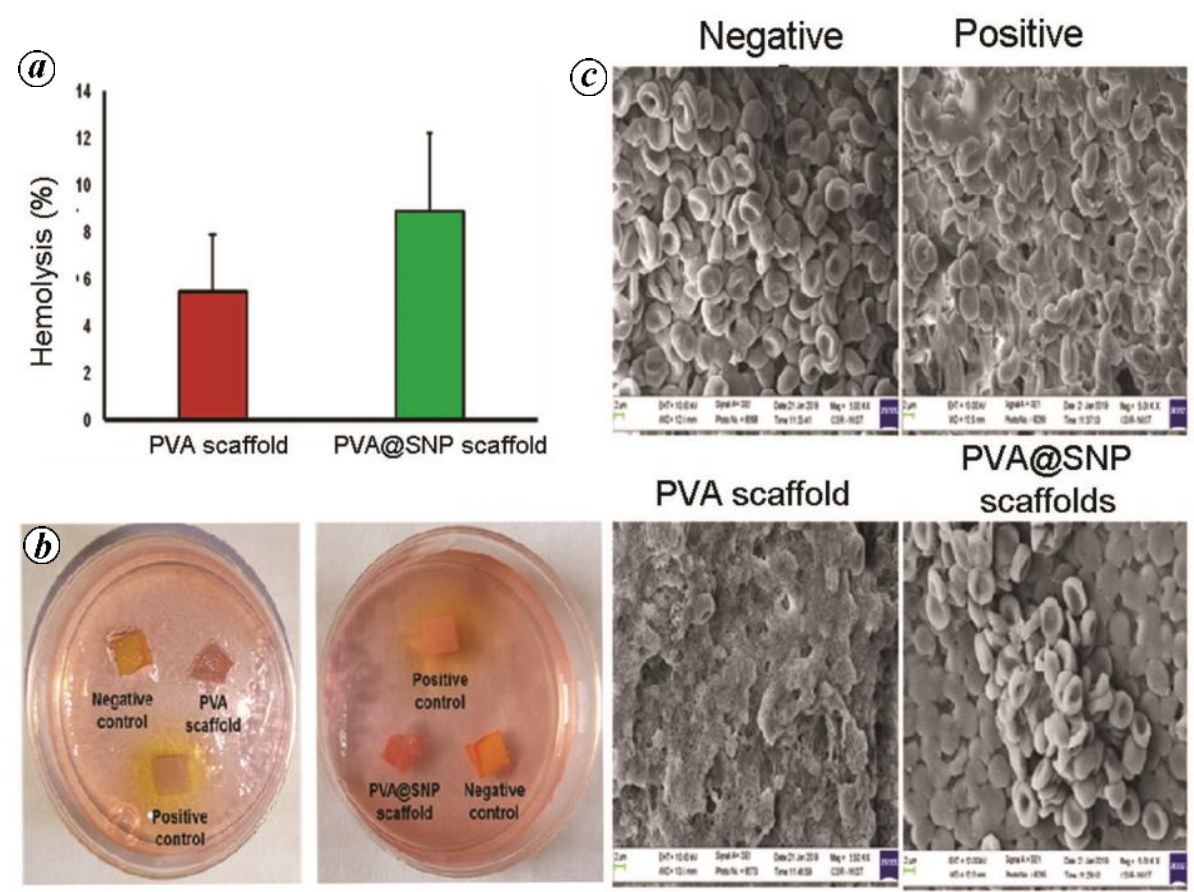

Figure 2. $\boldsymbol{a}$, Haemolysis activity evaluated on isolated red blood cell (RBC) on PVA and PVA@SNP scaffolds. $\boldsymbol{b}$, Images of agar diffusion plates showing clearing zone for positive control and no effects on negative and test samples. $\boldsymbol{c}$, Representative scanning electron microscope (SEM) images of RBC. Data represent mean \pm SD.
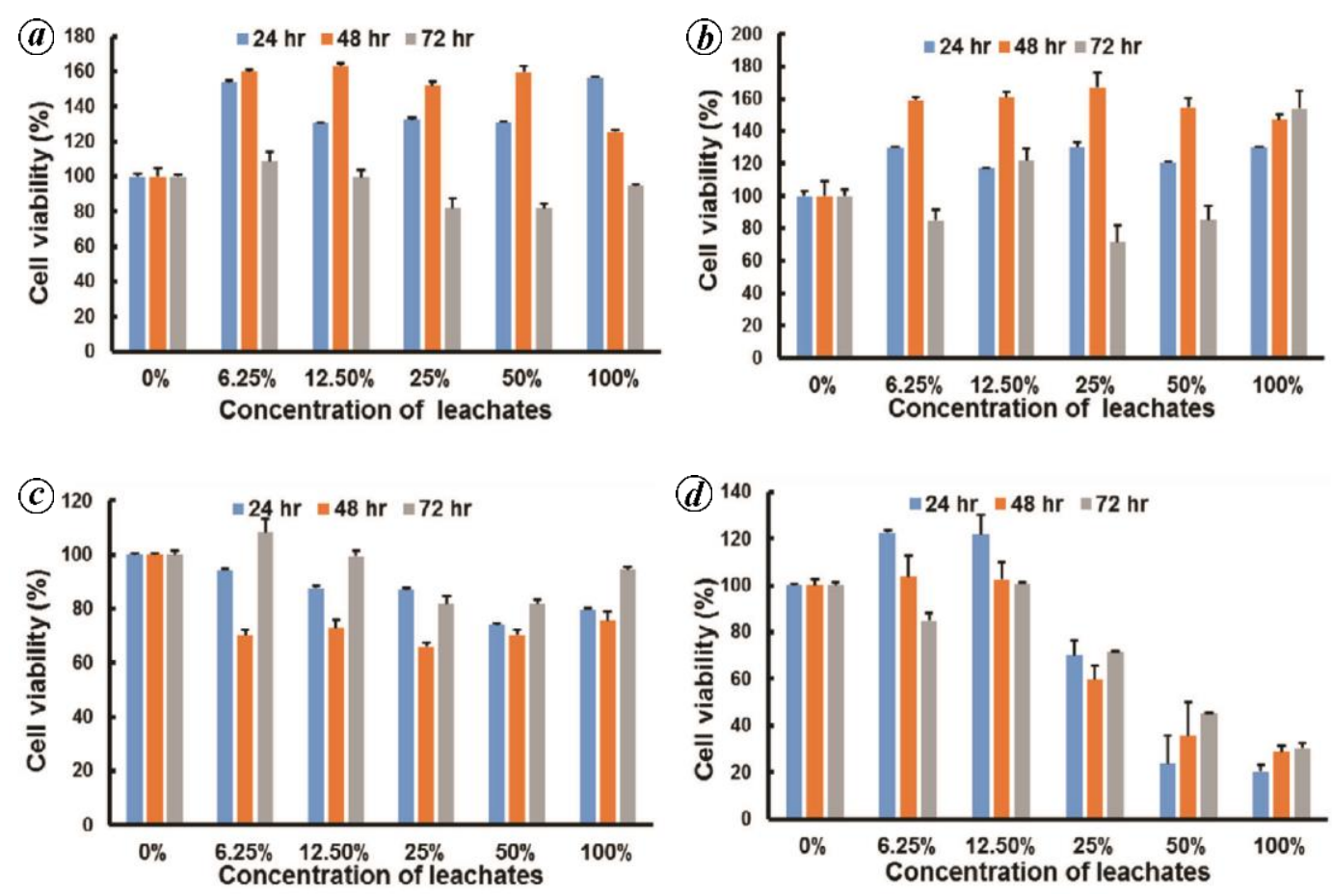

Figure 3. Indirect 3-(4,5-dimethylthiazol-2-yl)-2,5-diphenyltetrazolium bromide (MTT) assay using leachates collected from PVA scaffolds on $(\boldsymbol{a}) \mathrm{HaCaT},(\boldsymbol{b})$ A549 cells and PVA@SNP scaffolds on $(\boldsymbol{c})$ HaCaT and $(\boldsymbol{d})$ A549 cells. Data represent mean \pm SD.

antimicrobial properties of SNP@PST have been wellestablished previously? ${ }^{7}$ Lyophilized SNP $(1 \mathrm{mg})$ were uniformly fixed in $1 \mathrm{ml}$ of PVA solution and electrospun as previously mentioned. From $1 \mathrm{ml}$ solution $50 \mathrm{mg}$ scaffold was synthesized. For every study scaffolds of $5 \mathrm{~mm}$ diameter were used. SEM analysis of the scaffolds 
revealed that the fibres are of 100-200 nm diameter range. When SNP@PST was added to the electrospinning mixture, the fibre diameters were maintained but due to the hygroscopic nature of the polysaccharide, the fibres were blended and contrast was increased after the addition of SNP@PST (Figure $1 c$ and $d$ ). The chemical interactions that could have occurred during the formation of the scaffolds were studied using FTIR spectroscopy (Supplementary Figure 2). PVA predominantly has a hydroxyl group and the broadband observed between 3550 and $3200 \mathrm{~cm}^{-1}$ is associated with the $\mathrm{O}-\mathrm{H}$ stretch from the intermolecular and intramolecular hydrogen bonds $^{7}$. However, the weaker bands near 1340 and $1390 \mathrm{~cm}^{-1}$ as well as weak absorption around $1448 \mathrm{~cm}^{-1}$ represent the symmetric frequencies of $\mathrm{AgNO}_{3}$ (refs 5, 14), from which SNP@PST is formed.

Since the initial encounter of any externally administered particle occurs with blood components, it is important to determine the effect on RBCs. Therefore, haemolysis assays were performed to determine the toxicity of scaffolds on RBCs (Figure $2 a$ ). The haemolysis ratio represents the extent of RBCs broken by the sample contacting with blood. Generally speaking, a haemolysis percentage below 5 is suited for biomaterials for medical applications ${ }^{15}$. The haemolytic value was around 5 for PVA scaffolds and below 9 for PVA@SNP scaffolds. The increased haemolysis rate of PVA@SNP might be due to the presence of silver nanoparticles. In general, the scaffolds were found to be lacking any toxicity toward RBCs and well-suited for biomedical applications. SEM showed intact RBC thus confirming the compatibility.

Toxicity evaluation in terms of size of the zone of decolourization and degree of cell lysis using agar diffusion test is considered to be the gold standard for testing biomaterials. Clearing zone (yellow) was observed around the positive control (Triton X-100), whereas no particular changes were observed around PVA and PVA@SNP scaffolds, showing the cytocompatibility of both the scaffolds (Figure $2 b$ ). The response index for the positive control was moderate to severe as it produced a clearing zone of $1 \mathrm{~cm}$ or above. No zones were obtained for test samples or negative controls.

Cytotoxicity of the scaffolds was evaluated on a cancer and a normal cell line by the widely used MTT method. Metabolic conversion of MTT has often been used to monitor the viability or metabolic activity of cell populations. It has also been described as a measure of cell number within a population. Both indirect and direct MTT assays were employed for the purpose. Using indirect MTT assay we can evaluate whether the degradation products from the scaffolds are toxic to the cells or not. PVA scaffolds as such did not produce any toxic effects on cancer (Figure $3 a$ ) or normal cells (Figure $3 b$ ), and higher cell proliferation was also observed. When SNP@PST were loaded to the scaffolds, the leachates from the scaffolds showed moderate toxicity on cancer cell line A549, and no severe toxicity on normal cell line HaCaT (Figure $3 c$ and $d$ ). Even though lower concentrations of PVA scaffolds cause good cell proliferation on $\mathrm{HaCaT}$ cells, this trend was not observable with PVA@SNP scaffolds. In general, a trend of concentration-dependent reduction in cell proliferation was observed with both the scaffolds. The observation that the degradation products of both the scaffolds are non-toxic to normal human cells could be a strong factor in governing the future development of this scaffold for human applications. However, moderate toxicity of PVA@SNP towards cancer cells could be beneficial for retarding tumour growth after local administration of the scaffold.

In direct MTT assay, the viability of cells is evaluated after their growth over the scaffolds. It is to be noted that both scaffolds do not promote the proliferation of cells at any concentration for day-one to day-seven. The growth of normal human keratinocytes was not greatly affected by both the scaffolds, but PVA@SNP were more toxic in comparison with PVA scaffolds (Figure $4 a$ ). It is to be noted that both the scaffolds displayed greater toxicity towards cancer cells, especially at lower incubation periods (Figure $4 \mathrm{~b}$ ). The increased cytotoxicity of PVA@SNP scaffolds towards cancer cells could be attributed to the presence of SNP@PST, which have been well demonstrated to be excellent cancer-targeted cytotoxic nanoparticles ${ }^{1}$. The preferential non-toxic nature of PST001 as a capping agent could be the potential reason

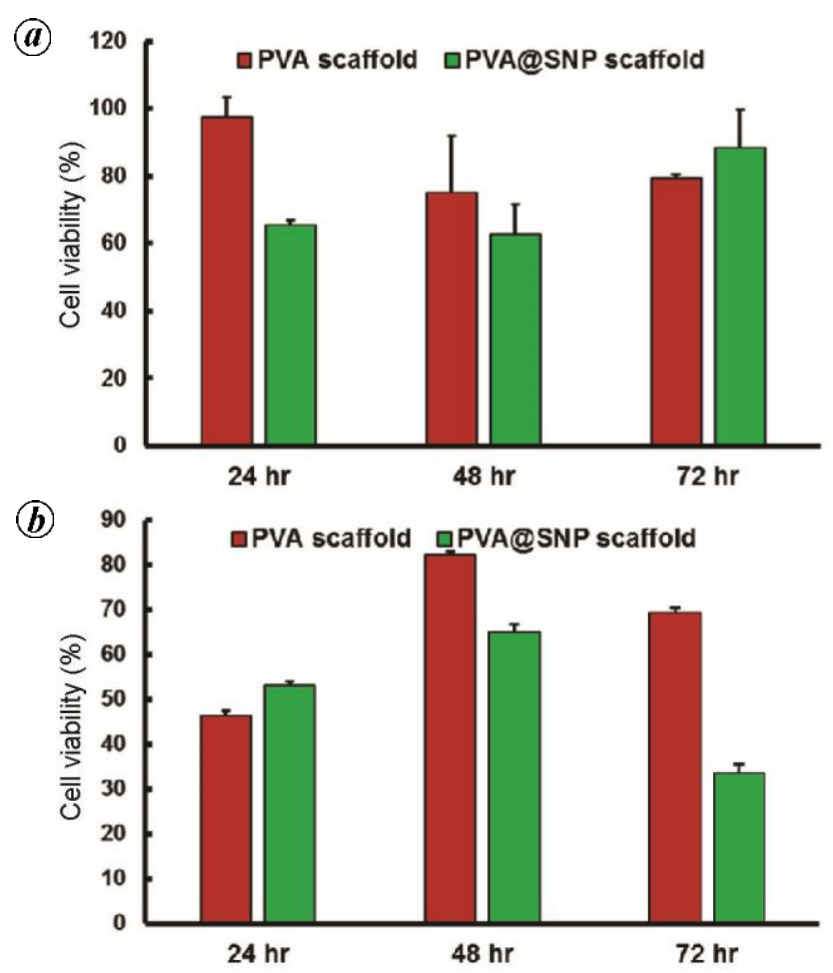

Figure 4. Direct MTT assay for the cytotoxic evaluation of PVA and PVA@SNP scaffolds on (a) HaCaT and (b) A549 cells under 24, 48 and $72 \mathrm{~h}$ of incubation. Data represent mean \pm SD. 


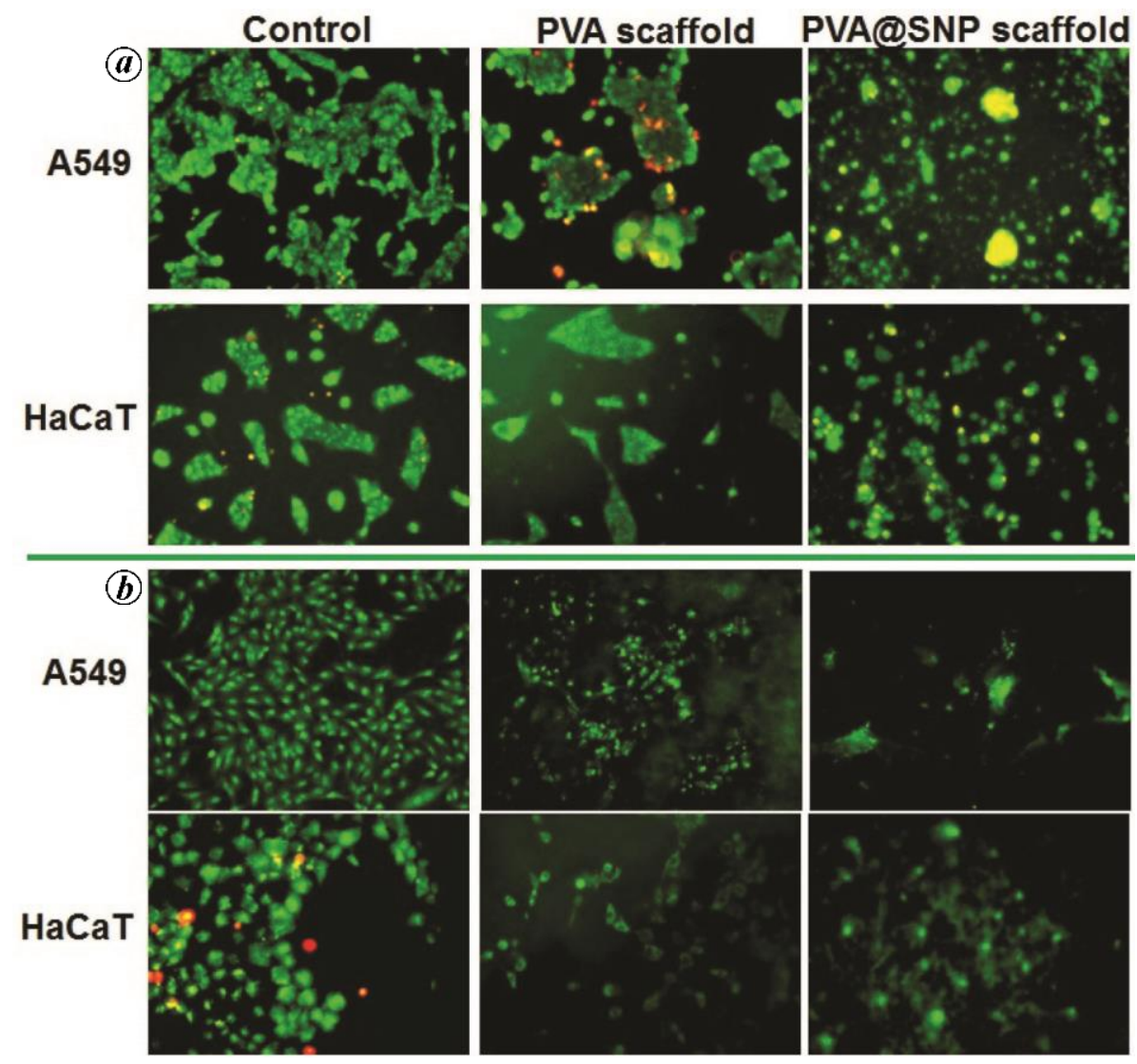

Figure 5. Indirect $(\boldsymbol{a})$ and direct $(\boldsymbol{b})$ live/dead imaging using acridine orange ethidium bromide staining on $\mathrm{A} 549$ and $\mathrm{HaCaT}$ cells.

E. coli
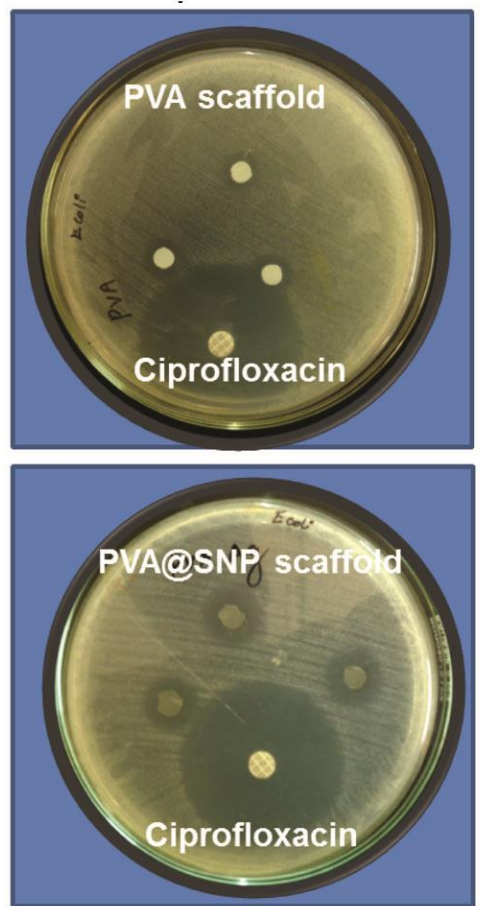

B. pumilus
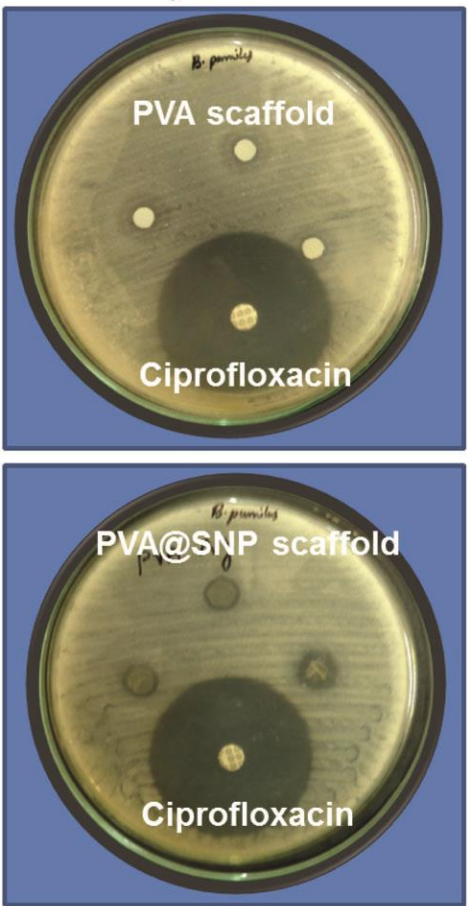

Figure 6. Agar diffusion assay for evaluating antibacterial activity of PVA and PVA@ SNP scaffolds using ciprofloxacin as positive control. 


\section{RESEARCH COMMUNICATIONS}

for the absence of any toxicity displayed by the nanoparticles towards normal cells. PVA has been previously reported and certified for use in biomaterial syntheses due to its non-toxicity to cells ${ }^{16}$

Two methods were employed for live/dead assay using the biocompatible scaffolds. For the indirect method, cells were seeded onto 96-well plates and leachates were treated after $24 \mathrm{~h}$ cell attachment. Green images indicate viable cells and orange/yellow images indicate dead cells. From these images, we can observe that the cancer cell A549 was dead after $24 \mathrm{~h}$ treatment and most of the cells were dead and floating, and washed-off during the imaging process. For the normal cells after PVA@SNP treatment, the cell morphology was changed but dead cells were much less compared to A549, but more compared to the PVA scaffold. In direct or contact live/dead staining, scaffolds were seeded with cells and the cells were imaged after $72 \mathrm{~h}$ incubation. The cells attached to the PVA scaffold displayed higher viability when compared with SNP-loaded scaffolds (Figure $5 a$ and $b$ ). The results observed with the indirect and direct live/dead staining were in good agreement with the cytotoxicity assays performed. In fact, the PVA scaffold could be highly non-toxic towards both cancer and normal cells, but addition of SNP@PST makes it moderately toxic towards cancer cells while being largely non-toxic towards normal human cells.

The well diffusion method was carried out to examine the antibacterial activity of scaffolds under in vitro conditions against E. coli and B. pumilus. Among the scaffolds, PVA@SNP displayed potential antimicrobial property against both the microbes when compared with the PVA scaffolds, indicating their efficacy as an antibacterial agent (Figure 6; Supplementary Table 2). The excellent antimicrobial activity of SNP@PST has been previously reported $^{5}$. The antibacterial property of PVA@SNP scaffolds could be attributed to the presence of SNP@PST, which could have been released from the scaffolds under optimal conditions.

Biocompatible scaffolds overcome most but not all problems associated with the cell-based two-dimensional monolayer cultures used as in vitro models, and hence open a new era in cancer management. In this study, 3D scaffolds fabricated using electrospinning, consisted of nanometre-sized random PVA fibres to mimic the orientation and size of extracellular matrix. The final design of the scaffold impregnated with silver nanoparticles, fabricated using a green chemistry approach, not only delivers excellent antibacterial effects but also makes it an effective anticancer material. Further in-depth biological studies are warranted to fully decipher the role of these hybrid scaffolds in tissue engineering.

1. Preethi, G., et al., Biomedical applications of natural polymer based nanofibrous scaffolds. Int. J. Med. Nano Res., 2015, 2(010), $1-9$
2. Sahoo, R., Sahoo, S. and Nayak, P., Release behavior of anticancer drug paclitaxel from tamarind seed polysaccharide galactoxyloglucan. Eur. J. Sci. Res., 2010, 47(2), 197-206.

3. Rao, P., Ghosh, T. and Krishna, S., Extraction and purification of tamarind seed polysaccharide. J. Sci. Ind. Res., 1946, 4, 705.

4. Aravind, S. et al., Antitumor and immunopotentiating activity of polysaccharide PST001 isolated from the seed kernel of Tamarindus indica: an in vivo study in mice. Sci. World J., 2012, 2012, 1-14.

5. Joseph, M. M. et al., Exploration of biogenic nano-chemobiotics fabricated by silver nanoparticle and galactoxyloglucan with an efficient biodistribution in solid tumor investigated by SERS fingerprinting. ACS Appl. Mater. Interfaces, 2017, 9(23), 19578 19590.

6. Supaphol, P. and Chuangchote, S., On the electrospinning of poly (vinyl alcohol) nanofiber mats: a revisit. J. Appl. Polym. Sci. 2008, 108(2), 969-978.

7. Park, J.-C. et al., Electrospun poly(vinyl alcohol) nanofibres: effects of degree of hydrolysis and enhanced water stability. Polymer J., 2010, 42, 273.

8. Destaye, A. G., Lin, C.-K. and Lee, C.-K., Glutaraldehyde vapor cross-linked nanofibrous PVA mat with in situ formed silver nanoparticles. ACS Appl. Mater. Interfaces, 2013, 5(11), 47454752.

9. Ramya, A. N. et al., Emergence of gold-mesoporous silica hybrid nanotheranostics: dox-encoded, folate targeted chemotherapy with modulation of SERS fingerprinting for apoptosis toward tumor eradication. Small, 2017, 13(31), 159-172.

10. Ignatius, A. and Claes, L. E., In vitro biocompatibility of bioresorbable polymers: poly (L, DL-lactide) and poly (L-lactideco-glycolide). Biomaterials, 1996, 17(8), 831-839.

11. Abdelgawad, A. M., Hudson, S. M. and Rojas, O. J., Antimicrobial wound dressing nanofiber mats from multicomponent (chitosan/silver-NPs/polyvinyl alcohol) systems. Carbohydr. Polym., 2014, 100, 166-178.

12. Joseph, M. M. et al., PST-gold nanoparticle as an effective anticancer agent with immunomodulatory properties. Colloids Surf. B, 2013, 104, 32-39.

13. Dasgupta, N. and Ramalingam, C., Silver nanoparticle antimicrobial activity explained by membrane rupture and reactive oxygen generation. Environ. Chem. Lett., 2016, 14(4), 477-485.

14. Devlin, J. P., Williamson, K. and Austin, G., Infrared spectrum of molten silver nitrate. J. Chem. Phys., 1966, 44(5), 2203-2204.

15. Zhou, C. and Yi, Z., Blood-compatibility of polyurethane/liquid crystal composite membranes. Biomaterials, 1999, 20(22), 2093 2099.

16. Koski, A., Yim, K. and Shivkumar, S., Effect of molecular weight on fibrous PVA produced by electrospinning. Mater. Lett., 2004, 58(3-4), 493-497.

ACKNOWLEDGEMENTS. We thank the Kerala State Council for Science, Technology and Environment (No. KSCSTE/5166/2017SRSHS dated 11/09/2017), Government of Kerala for financial support and University Grants Commission, New Delhi for providing research fellowships to G.U.P., B.S.U. and R.S.

Received 13 November 2018; revised accepted 23 February 2019

doi: $10.18520 / \mathrm{cs} / \mathrm{v} 116 / \mathrm{i} 10 / 1735-1741$ 